\title{
Dynamic monitoring of a migrating and penetrating intracardiac foreign body by echocardiography perioperatively
}

\section{HJ Huang}

Changzheng Hospital

\section{S Zhang}

Changzheng Hospital

\section{P Wang}

Changzheng Hospital

XF Ji

Changzheng Hospital

\section{ZN Wang}

Changzheng Hospital

jiaqi zhao ( $\nabla$ czzjq@outlook.com )

Changzheng Hospital https://orcid.org/0000-0002-9465-8086

\section{Case report}

Keywords: foreign body, echocardiography, monitor, cardiac, pericardial effusion

Posted Date: January 24th, 2020

DOl: https://doi.org/10.21203/rs.2.21796/v1

License: (1) This work is licensed under a Creative Commons Attribution 4.0 International License. Read Full License 


\section{Abstract}

Backgroud: Cardiac penetrating injuries caused by migrating foreign bodies are rare. The preoperative and intraoperative locating of these foreign bodies is challenging since their location may change with the continuous beat of the heart.

Case presentation: We reported a case of cardiac penetrating injury caused by a self-inflicted needle that migrated from the neck to the heart. The needle was dynamically monitored by both transthoracic echocardiography (TTE) and transesophageal echocardiography (TEE) perioperatively, which showed a significant location change of the needle in the ventricular wall. The migrating distance of the needle moving into the right ventricle was about $2.0 \mathrm{~cm}$. The dynamic monitoring of the needle's location using echocardiography helped clinical decisions and benefited the patient.

Conclusions: Foreign bodies with sharp nature have a tendency to migrate though tissues and lead to serious complications. Echocardiography with a combination of both TTE and TEE has a unique advantage in providing real-time, continuous and accurate monitoring of cardiac foreign bodies. It helps to determine optimal surgical method without radiation hazards, which is worth popularizing in clinical practice.

\section{Backgroud}

Cardiac penetrating injuries caused by migrating foreign bodies are rare, which usually need early diagnosis and prompt surgical managements. However, due to the continuous beat of the heart, it is difficult to accurately locate the intracardiac foreign bodies both preoperatively and intraoperatively ${ }^{1}$. Especially the foreign bodies may migrate through the myocardial tissues along with the systolic and diastolic beats of the heart, resulting in potential location changes and life-threatening consequences, such as cardiac tamponade, pneumothorax, etc. Therefore, a dynamic monitoring of the foreign bodies is clinically required in that it could facilitate the real-time localization of the foreign bodies and the choice of operative approaches. Here, we provided an unusual case of a migrating and penetrating intracardiac needle from the neck to the heart, which was dynamically monitored by both transthoracic echocardiography (TTE) and transesophageal echocardiography (TEE) perioperatively.

\section{Case Presentation Chief complaints}

A 49-year-old man was admitted to the emergency department with chest pain, which had been progressively worsened during the past 32 hours.

\section{History of present illness}


One year ago, the patient had penetrated a needle into his left neck after drinking alcohol. Six months later, the X-ray examination showed a metal density shadow in the left neck, which was left untreated (Fig. 1). There had been no special discomfort for the patient during the past year until the onset.

\section{Physical examination}

Vital signs were stable except for the tachycardia and tachypnoea. The patient's heart rate was $113 \mathrm{bpm}$, the respiratory rate was 27 breaths per minute, the temperature was $36.5^{\circ} \mathrm{C}$, and the blood pressure was $122 / 89 \mathrm{mmHg}$. The auscultation of heart sounds was distant. There were no other positive findings on physical examinations.

\section{Laboratory and Imaging examinations}

Laboratory tests revealed a slightly elevated white blood cell count $\left(10.0 \times 10^{9} / \mathrm{L}\right)$, normal red hemoglobin levels, normal platelet count and normal levels of myocardial enzymes. Electrocardiogram (ECG) revealed sinus tachycardia and ST segment elevation in the precordial leads I,II, aVF, aVL, and V4-V6. Chest CT showed a linear metal density shadow in right ventricle, with suspected pericardial effusion (Fig. 2).

\section{Diagnosis and treatment process}

(1)

Preoperative diagnosis Although the chest CT showed a linear metal density shadow in right ventricle, the cardiothoracic surgeons could not definitely determine whether the stump of the foreign body was in the pericardial cavity or had completely entered the heart. This was important for the choice of surgical method. To accurately locate the foreign body, a bedside TTE (Philips, EPIQ7C, S5-1,9 Hz) was immediately performed. There was an obvious pericardial effusion in anterior and lateral pericardium, with a deepest value of $1.5 \mathrm{~cm}$ at the anterolateral wall of the right ventricle (Fig. 3). In the parasternal short-axis view, we found a line-like strong echo in the right ventricle. Though the line-like strong echo was similar to that of the right ventricular chordae, it penetrated the right ventricular wall and extended into the pericardial cavity. Thus, we speculated that the line-like strong echo was probably the target foreign body (Fig. 4). The length of the line-like strong echo was about $0.2 \mathrm{~mm}$ in the right ventricle and $2.5 \mathrm{~cm}$ in the pericardial cavity. There were no other positive findings from TTE: normal left ventricular systolic function, normal pulmonary artery pressure, no segmental wall motion abnormalities, no intracardiac shunts and no signs of significant reflux.

(2)

Intraoperative detection The patient was then admitted for cardiac foreign body removal surgery. By means of intraoperative TEE (Philips, EPIQ7C, X7-2t,53 Hz) detection in the operating room, we found that the line-like strong echo was in the lower posterior wall of the right ventricle, near the left lobe of liver. Compared with bedside TTE which was performed 2 hours ago, there was significantly increased pericardial effusion, with a deepest value of $2.3 \mathrm{~cm}$ at the anterior pericardium (Fig. 5). Importantly, the line-like strong echo moved significantly towards the heart. The length of the line-like strong echo was 
about $1.0 \mathrm{~cm}$ in the pericardial cavity, leaving a longer part of the strong echo in the right ventricle (Fig. 6a, b). Twenty minutes later, TEE was reapplied to display a shorter length of the line-like strong echo in the pericardial cavity, for about $0.6 \mathrm{~cm}$ (Fig. 7).

The operation was then immediately performed without cardio-pulmonary bypass(CPB). After cutting the pericardium and subsequent pericardial suspension, about $300 \mathrm{ml}$ bloody liquid was drainaged from the pericardial cavity. The extracardiac exploration revealed flocculent fibrin exudation on the surface of the visceral pericardium, with the lower posterior surface of the right ventricle the most prominent. A fine metal needle was found beneath the fibrin, with a length of only $0.3 \mathrm{~cm}$ outside the right ventricle wall (Fig. 8a). The needle was pulled out quickly with a full length of $3.0 \mathrm{~cm}$ (Fig. 8b). In addition, there was a $3.0 \times 3.0 \mathrm{~cm}$ laceration in the diaphragm opposite the needle tip (Fig. 9). After removal of the needle, the right ventricle wound received purse-string suture and the diaphragm was repaired.

(3)

Postoperative evaluation The patient's vital signs were stable. Bedside TTE and Chest CT showed no abnormality: no signs of foreign body, no intracardiac shunts, normal left ventricular systolic function, and normal functions of each valve. The patient was discharged after an uneventful recovery.

\section{Discussion And Conclusions}

Generally, foreign bodies may reach the heart via direct penetration due to local trauma or through intravenous migration after certain medical procedures, such as catheter pieces, pacemaker electrodes and stents ${ }^{2,3}$. Darko et al. reported a case of Kirschner wire moving from right hip to right ventricle after an orthopedic surgery ${ }^{4}$. Foreign bodies with sharp nature have a tendency to migrate though tissues and lead to serious complications. Wang et al. reported a case that a swallowed fish bone resulting in cardiac injury and devastating tamponade ${ }^{5}$. In the present case, there was a risk that the needle might penetrate the diaphragm into the abdominal cavity, which would probably cause abdominal organ damage and bleeding. Once in the abdominal cavity, it would be more difficult to detect the fine needle surgically. Another, the needle might enter the right ventricle and injure the tricuspid valve, ventricular septum, or pulmonary artery, leading to valvular reflux, ventricular septal defect, infective endocarditis or thrombosis ${ }^{6}$. In addition, the increasing pericardial effusion might cause devastating tamponade. The clinicians should be alert that the foreign bodies with sharp nature could migrate though tissues fast with serious consequences. The dynamic monitoring of the foreign bodies' location is urgently required.

According to our experience and cognition, a self-inflicted foreign body migrating from peripheral tissues to the heart was uncommon and rarely reported. Also, this is a rare report to record the dynamic movement of the foreign body in the myocardium perioperatively.

Preoperative localization of the foreign body was crucial for its surgical removal. X-rays, CT scans, fluoroscopy, and echocardiography were commonly used to locate the foreign body in the heart ${ }^{7}$. Studies showed that TTE provides information regarding the size, and location of cardiac foreign bodies with 
almost $100 \%$ sensitivity ${ }^{8}$. Moreover, TEE is a favorable imaging modality in assessing functional and structural damage of the heart, especially in the unstable patient ${ }^{9}$. It was important to note that the foreign body may migrate with blood flow, gravity, or through the myocardial tissues along with the systolic and diastolic beat of heart, resulting in location changes. Up to now, most operative suites lack real-time CT or magnetic resonance imaging (MRI). Kumar et al. reported a case in which the preoperative radiological imaging showed a foreign body in the posterior mediastinum of a blast-injury victim ${ }^{7}$. However, the foreign body could not be located in the area identified preoperatively during the extended right posterolateral thoracotomy. Interestingly, the intra-operative TEE showed it in the left atrium, which later migrated into the left ventricle necessitating a change in surgical approach for its removal.

In the present case, it was infered that the speculated migrating path of the needle was as follows. The needle rested on the left neck for at least 6 months before it penetrated into the peripheral vein. Thus, the needle migrated, by both blood flow and gravity, to the superior vena cava, right atrium, and then settled in right ventricle tentatively. With the continuous beats of heart, the needle tip penetrated the anterolateral wall of the right ventricle, and injured the diaphragm nearby. Fortunately, the needle did not penetrate the diaphragm or detach from the heart. In this process, the pericardial effusion developed due to the injured small myocardial vessels. Then the needle moved back towards the right ventricle, the process of which was captured by echocardiography. In a few hours, the length of the needle in the pericardial cavity reduced from $2.5 \mathrm{~cm}$ to $0.5 \mathrm{~cm}$, indicating that the migrating distance of the needle moving into the right ventricle was about $2.0 \mathrm{~cm}$. An emergency surgery was immediately carried out and the needle was successfully removed without open heart surgery and cardiopulmonary bypass (CPB). If there had been any delay, it would be possible that the needle might enter the right ventricle and was not visible on the surface of the visceral pericardium. In that case, the patient would have to undergo open heart surgery. The dynamic monitoring of the needle's location using echocardiography helped clinical decisions and benefited the patient.

In conclusion, echocardiography with a combination of both TTE and TEE has a unique advantage in providing real-time, continuous and accurate monitoring of cardiac foreign bodies to help determination of optimal surgical method without radiation hazards, which is worth popularizing in clinical practice.

\section{Declarations}

\section{Acknowledgements}

Not applicable.

\section{Consent for publication}

Written informed consent for publication of clinical data and clinical images was obtained from the patient. 
Not applicable.

\section{Authors' contributions}

Huang $\mathrm{HJ}$ and Zhang S made substantial contributions to the study conception and design and writing. Wang $\mathrm{P}$ and Ji XF provided relevant figures and were involved in drafting the manuscript. Zhao JQ and Wang ZN critically revised the manuscript for important intellectual content. All authors read and approved the final manuscript.

\section{Availability of data and materials}

Data and materials sharing were applicable to this article.

\section{Funding}

This work was supported by grants from the National Natural Science Foundation of China (Grant No. 81501492₫81602617) and Pyramid Talent Project of Changzheng Hospital.

\section{Competing interests}

The authors declare that they have no competing interests.

\section{References}

1.

Wang X, Zhao X, Du D. Management of Metallic Foreign Bodies in the Heart. J Card Surg. 2012;27(6):704-6.

2. Marina, Leitman MD. Foreign Bodies in the Heart Echocardiography. 2015;32(2):365-71. 3. Actis Dato GM, Arslanian A, Di Marzio P. Posttraumatic and iatrogenic foreign bodies in the heart: report of fourteen cases and review of the literature. J Thorac Cardiovasc Surg. 2003;126(2):408-14. 4.

Anić D, Brida V. I Jelić. The cardiac migration of a Kirschner wire: A case report. Texas Heart Institute journal / from the Texas Heart Institute of St. Luke's Episcopal Hospital, Texas Children's Hospital. 1997;24(4):359-361.

5.

Wang QQ, Hu Y, Zhu LF. Fish bone-induced myocardial injury leading to a misdiagnosis of acute myocardial infarction: A case report. World J Clin Cases. 2019;7(20):3335-40.

6. Supomo DH. An Unusual Foreign Body in the Heart: A Case Report. Ann Thorac Cardiovasc Surg. 2018;24(4):205-7.

7. 
Kumar B, Badamali AK, Jayant A. Intraoperative localization and monitoring of migrating foreign body using transesophageal echocardiography. Annals of Cardiac Anaesthesia. 2014;17(4):314-7.

8.

Dato GMA, Arslanian A, Marzio PD, et al. Posttraumatic and iatrogenic foreign bodies in the heart: Report of fourteen cases and review of the literature. J Thorac Cardiovasc Surg. 2003;126(2):408-14.

9.

Heldmann MG, Martin AK, Hebert J. Localization of Missile Tract and Intrapericardial Foreign Body with Computed Tomography: Case Report and Review of the Literature. The Journal of Trauma: Injury Infection Critical Care. 2006;60(2):410-3.

\section{Figures}




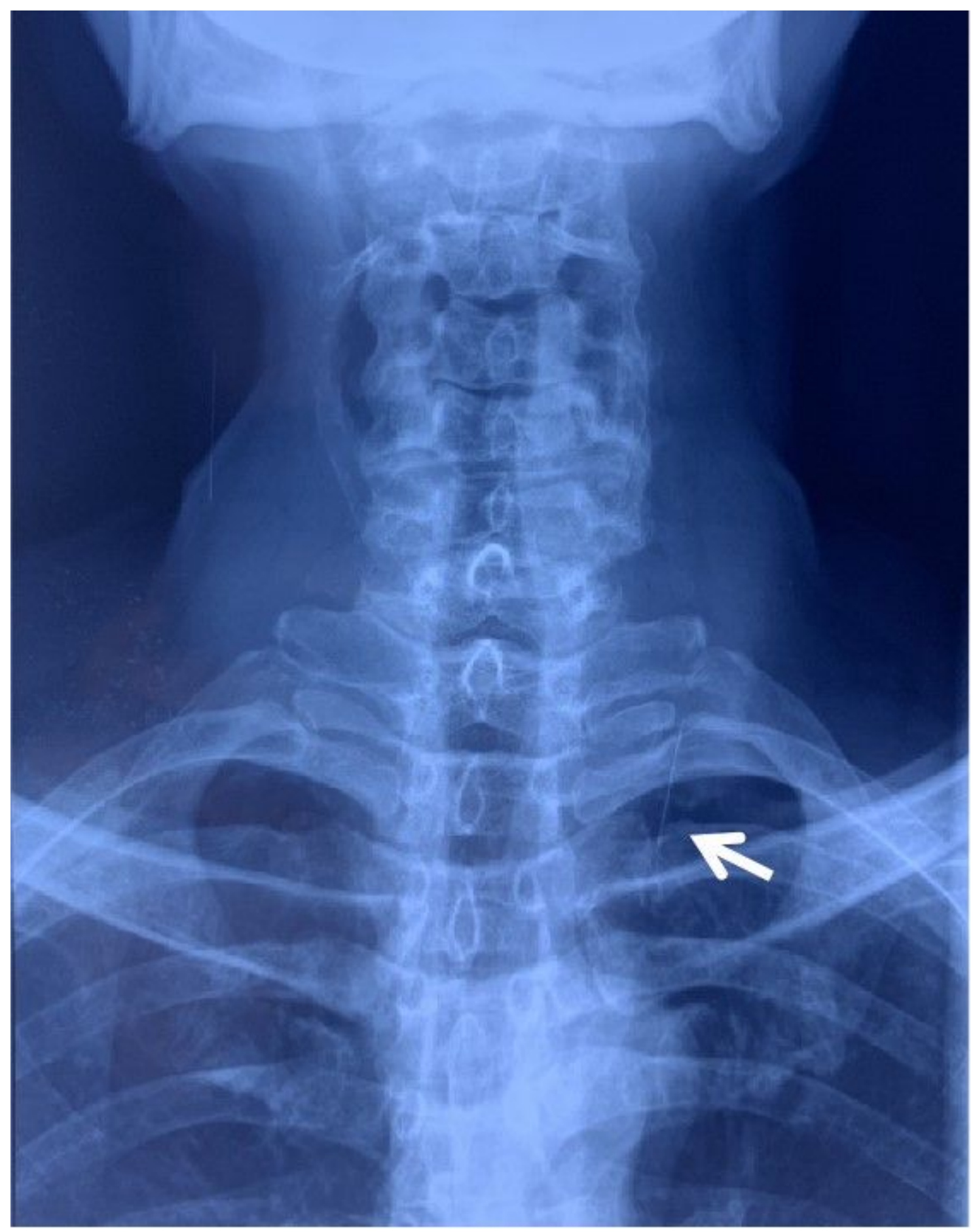

Figure 2

The X-ray examination showed a metal density shadow in the left neck of the patient. The arrow indicated the metal density shadow. 


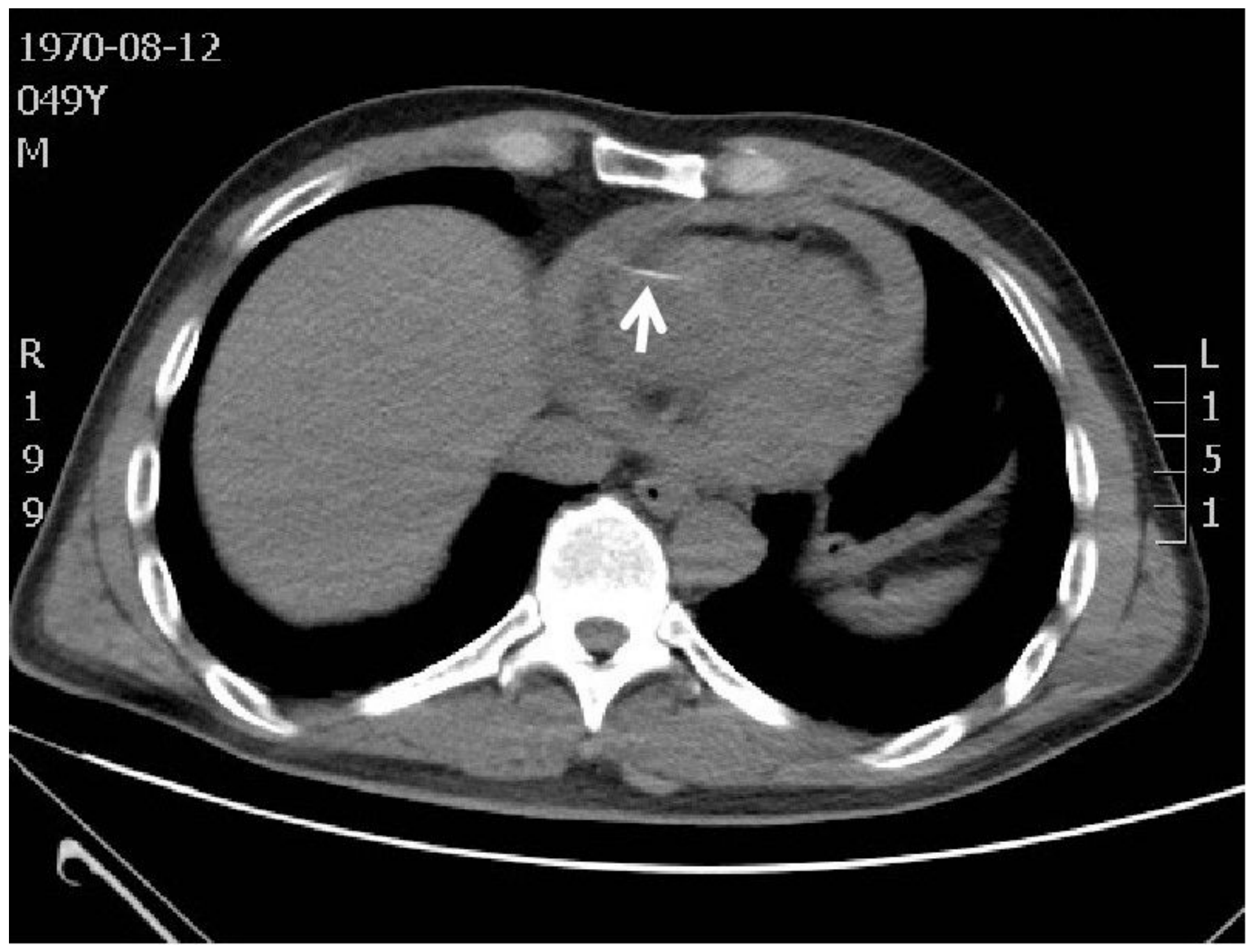

Figure 4

The chest CT showed a linear metal density shadow in right ventricle, with suspected pericardial effusion. The arrow indicated the linear metal density shadow. 


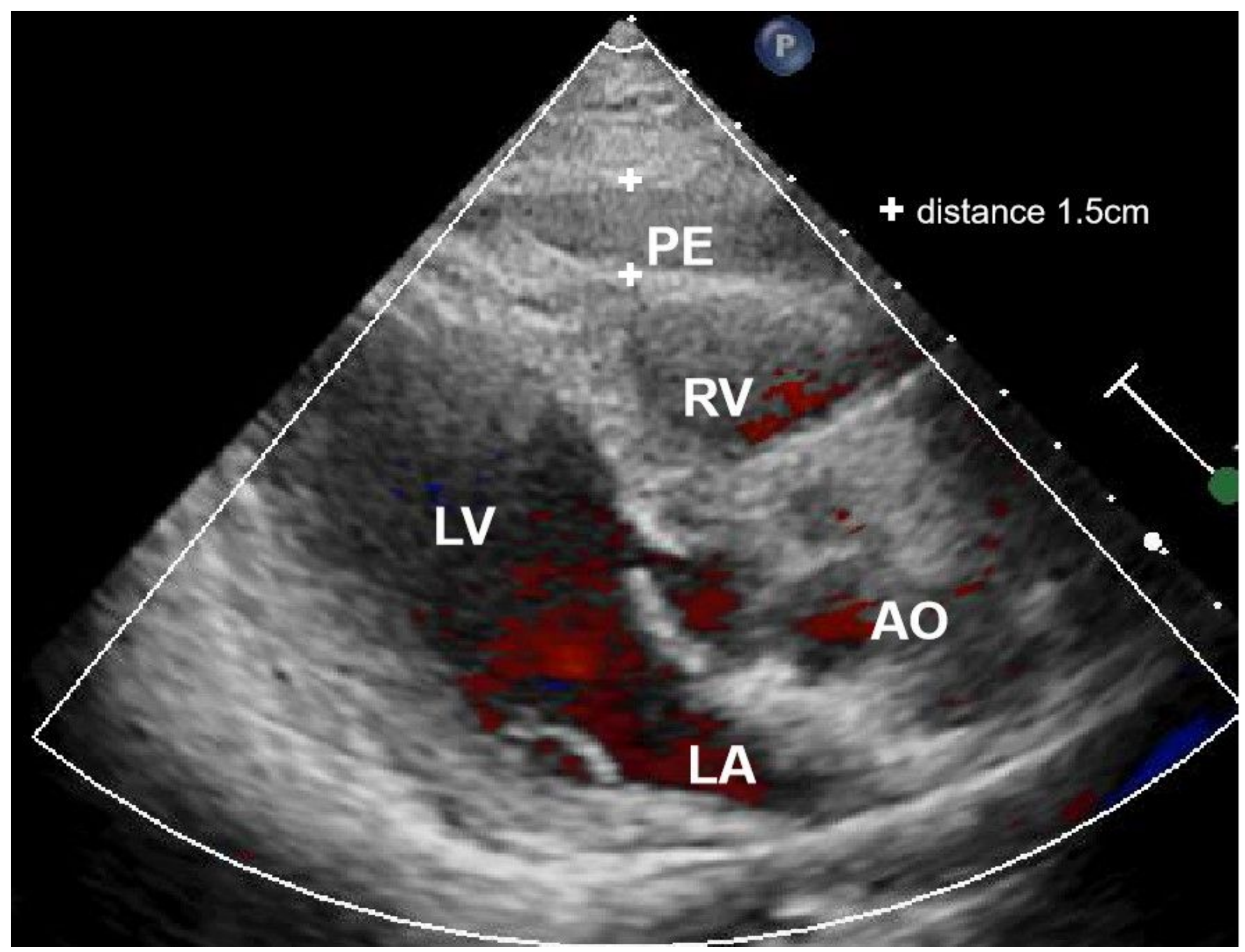

Figure 6

The parasternal long-axis view during TTE showed an obvious pericardial effusion in anterior and lateral pericardium, with a deepest value of $1.5 \mathrm{~cm}$ at the anterolateral wall of the right ventricle. PE: pericardial effusion; AO: aorta; LA: left atrium; LV: left ventricle; RV: right ventricle. 


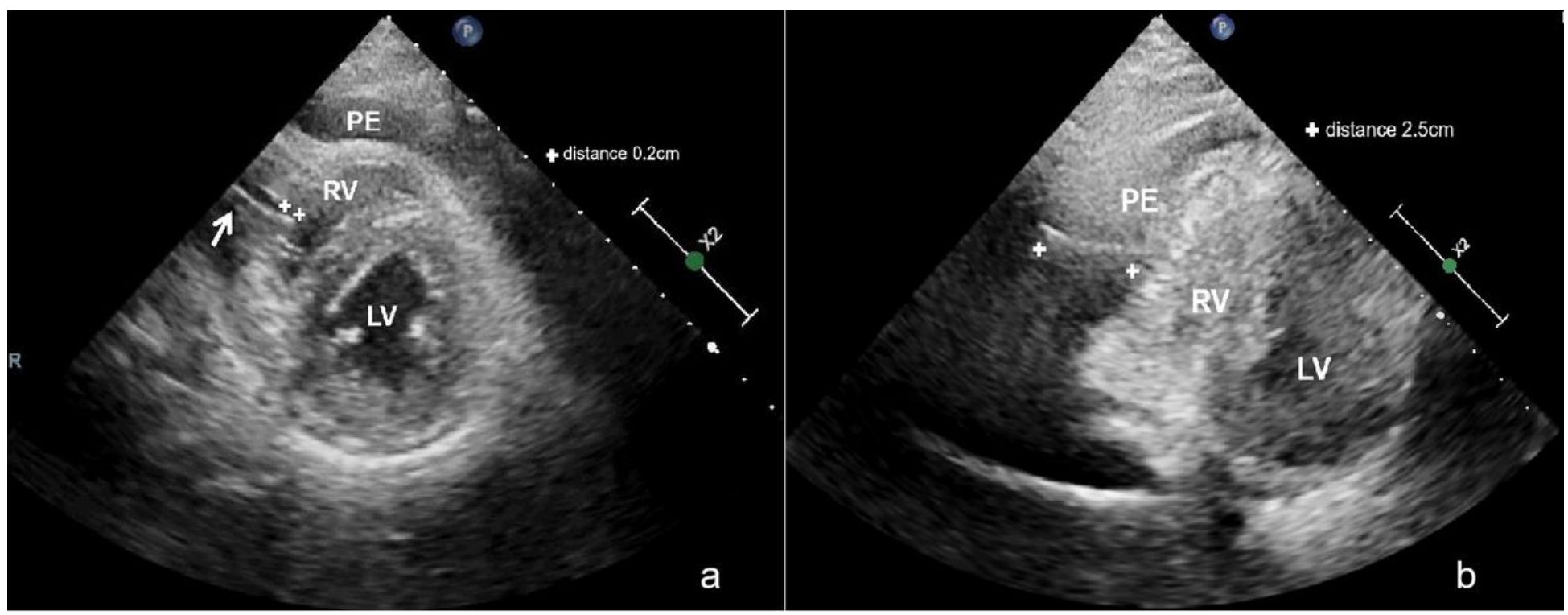

\section{Figure 8}

The parasternal short-axis view during TTE showed a line-like strong echo in the right ventricle. The linelike strong echo penetrated the right ventricular wall and extended into the pericardial effusion. The length of the line-like strong echo was about $0.2 \mathrm{~mm}$ in the right ventricle (4a) and $2.5 \mathrm{~cm}$ in the pericardial effusion (4b). LV: left ventricle; RV: right ventricle;PE: pericardial effusion. 


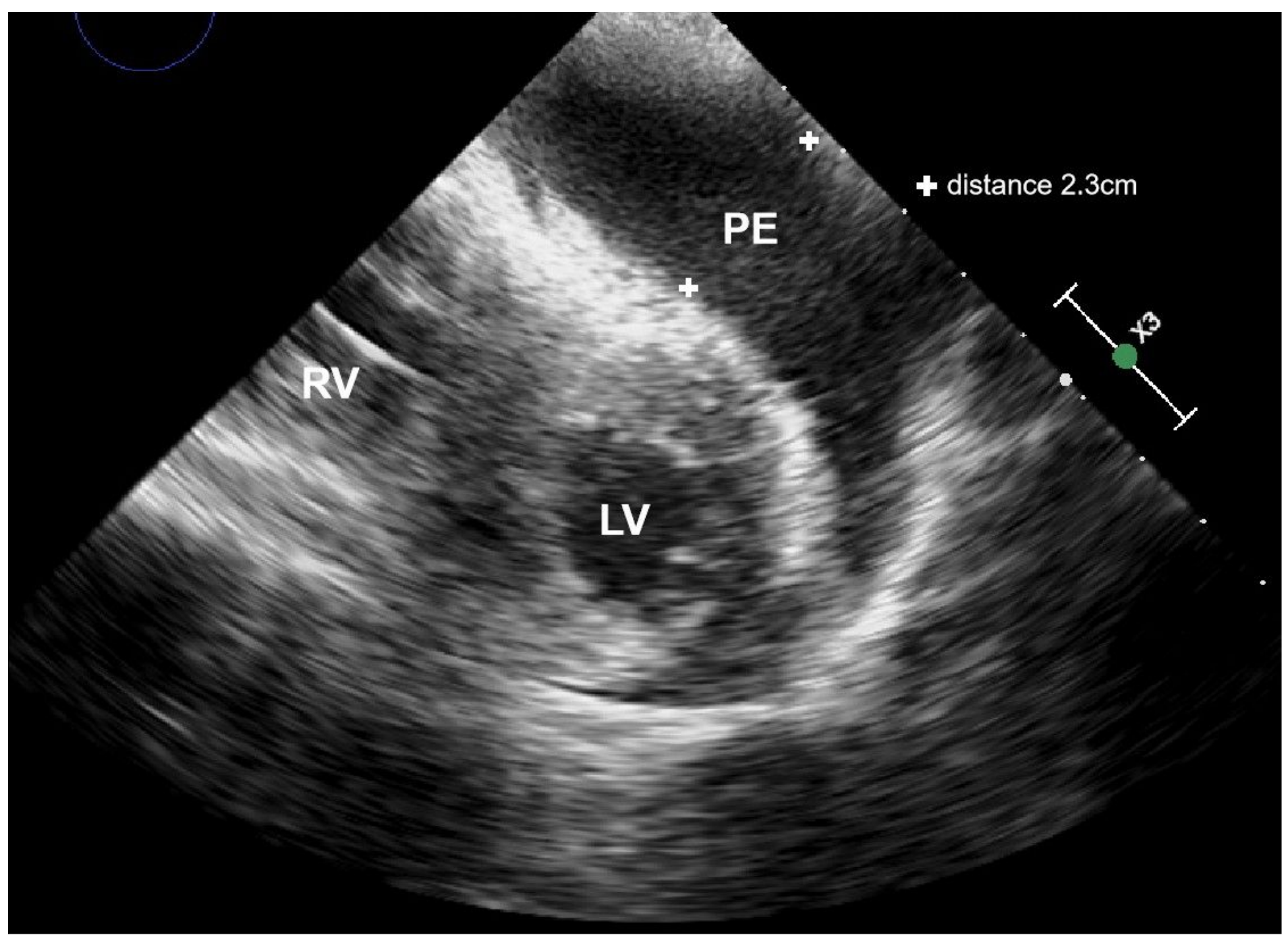

Figure 10

TEE showed significantly increased pericardial effusion, with a deepest value of $2.3 \mathrm{~cm}$ at the anterior pericardium. LV: left ventricle; RV: right ventricle;PE: pericardial effusion.

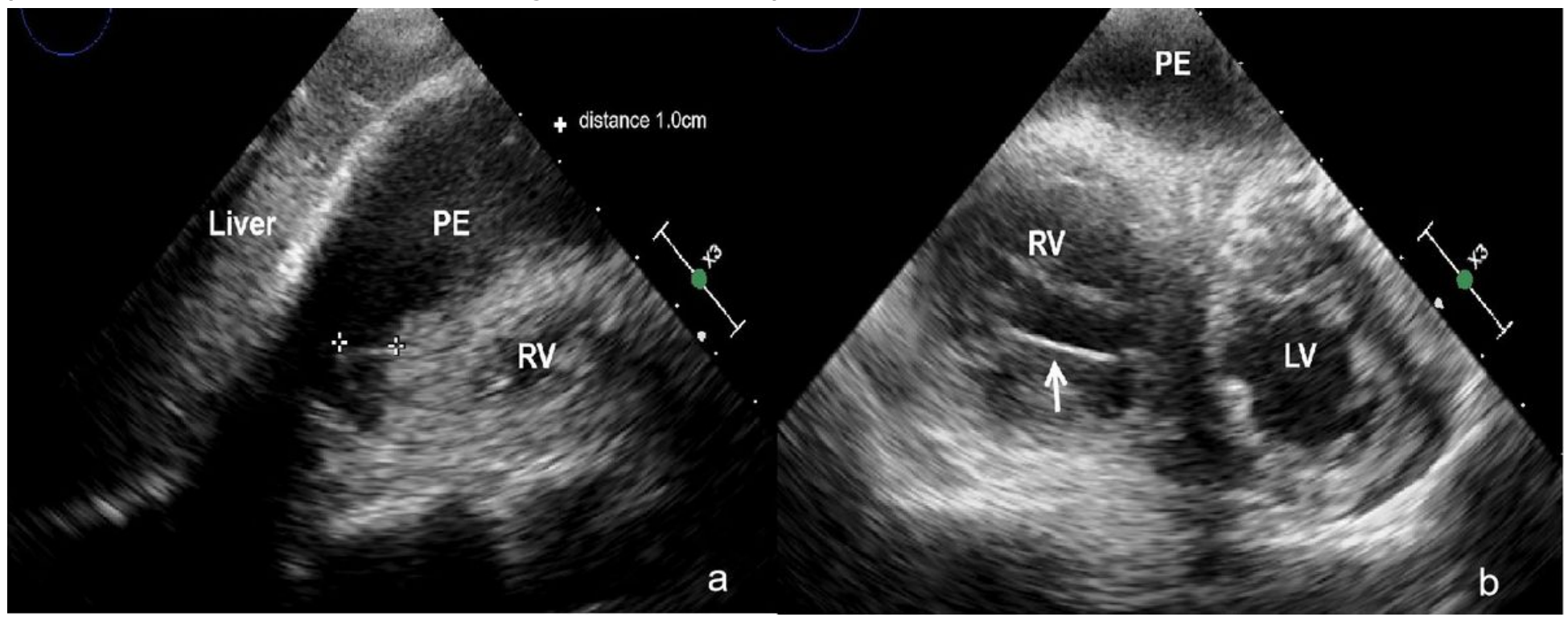


Figure 12

TEE showed that the line-like strong echo moved significantly towards the heart. The length of the linelike strong echo in the pericardial effusion was about $1.0 \mathrm{~cm}(6 a)$. The majority of the strong echo was in the right ventricle (6b). The arrow indicated the line-like strong echo in the right ventricle. LV: left ventricle; $\mathrm{RV}$ : right ventricle;PE: pericardial effusion.

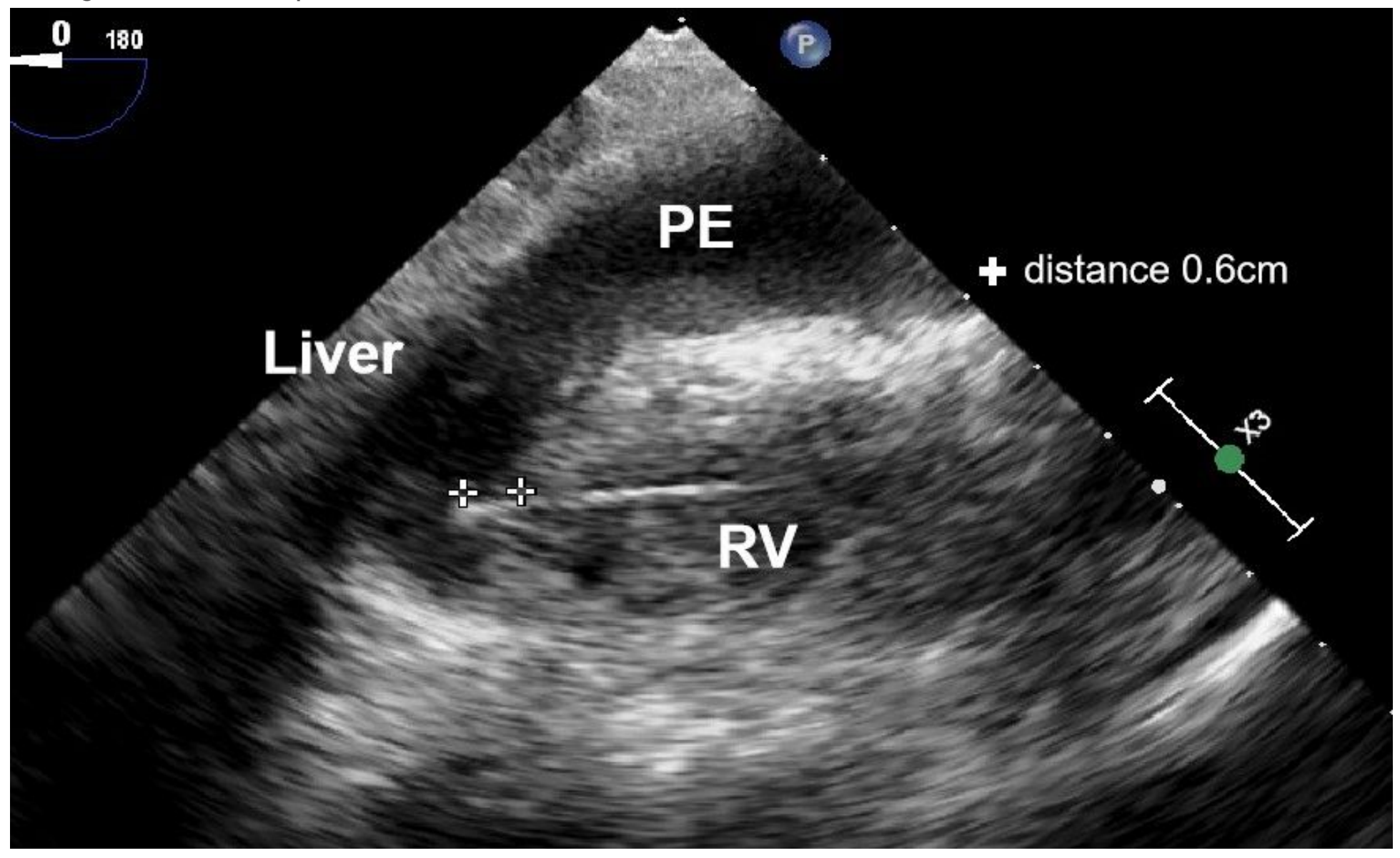

Figure 13

TEE showed that length of the line-like strong echo in the pericardial effusion was about $0.6 \mathrm{~cm}$. RV: right ventricle;PE: pericardial effusion. 


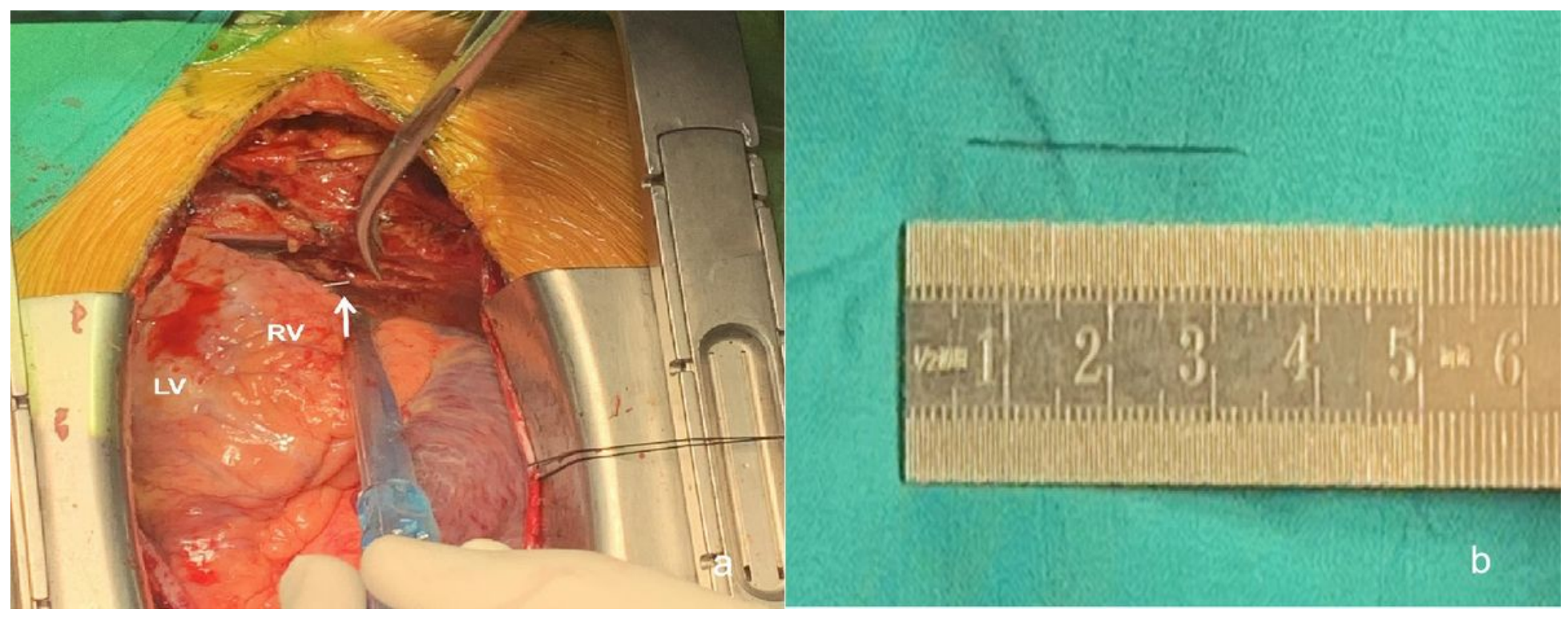

\section{Figure 15}

During extracardiac exploration, the stump of a fine metal needle was found on the lower posterior surface of the right ventricle, with a length of only $0.3 \mathrm{~cm}$ outside the right ventricle wall (Figure 8a). The full lenth of the needle was about $3.0 \mathrm{~cm}$ (Figure 8b). LV: left ventricle; RV: right ventricle. The arrow indicated the the stump of the needle. 


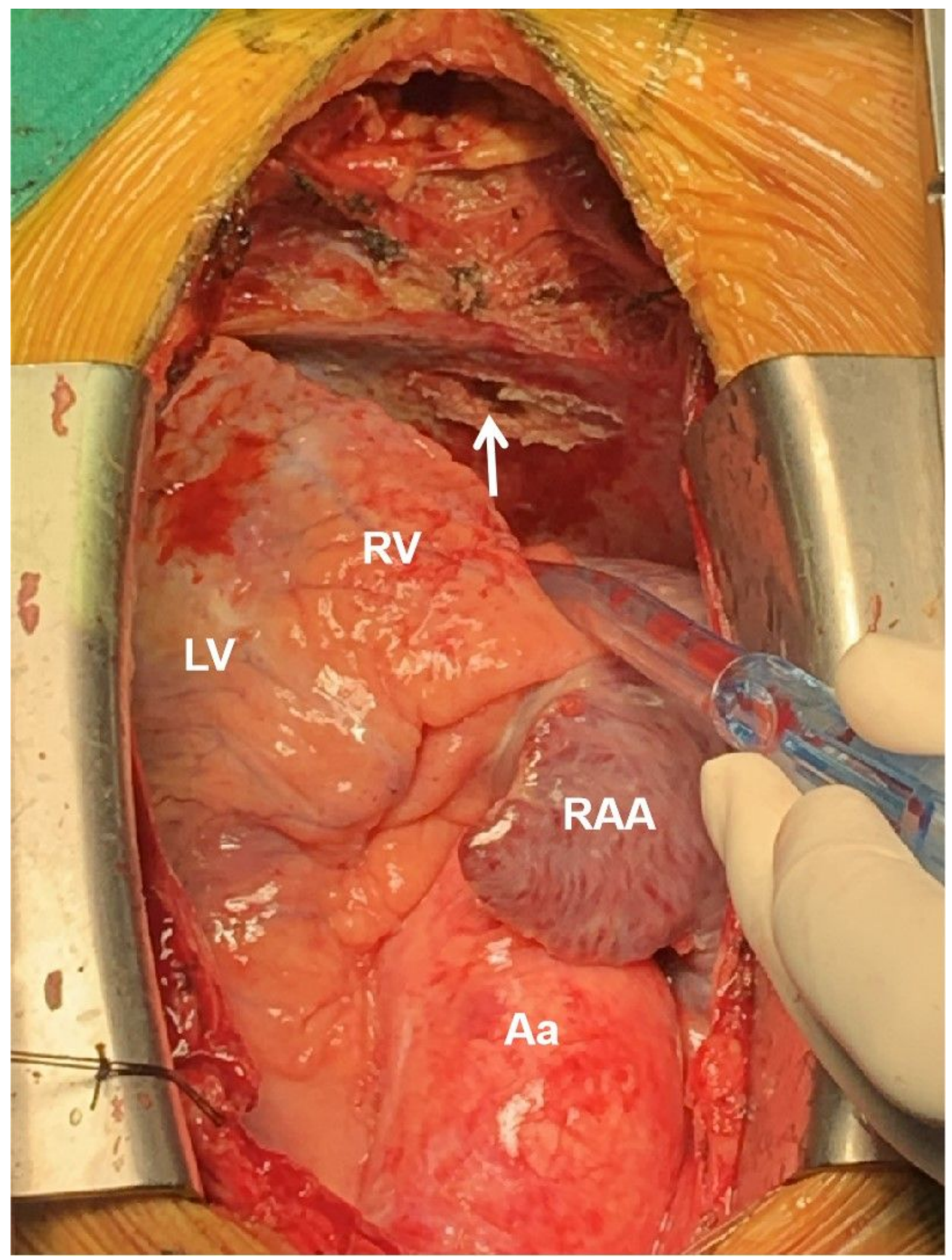

\section{Figure 18}

: In the diaphragm there was a 3.0x3.0cm laceration opposite the needle tip. Aa: ascending aorta; Raa: right atrial appendage; LV: left ventricle; RV: right ventricle. The arrow indicated the laceration in the diaphragm. 